\title{
Advancing the understanding of progression in multiple sclerosis: an interview with Shibeshih Belachew
}

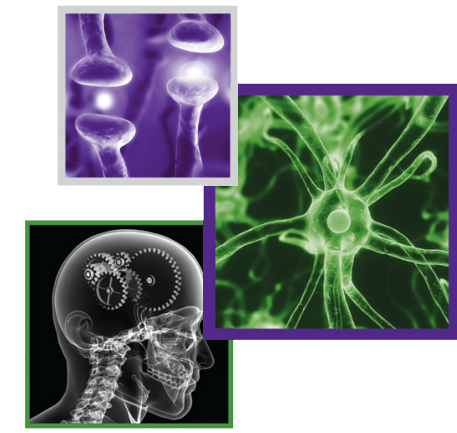

\author{
Shibeshih Belachew*,1 \\ ${ }^{1}$ Senior International Medical Director for Global Product Development Medical Affairs at F Hoffmann-La Roche Ltd, Basel, \\ Switzerland \\ * Author for correspondence: shibeshih.belachew@roche.com
}

\begin{abstract}
Shibeshih Belachew* speaks to Laura Dormer, Commissioning Editor: Dr Shibeshih Belachew, MD, PhD, is a Senior Medical Director for Multiple Sclerosis (MS) Disease Area in Global Product Development Medical Affairs at Roche (Basel, Switzerland). Prior to joining Roche in January 2016, he was Director of MS Franchise and Head of Medical Director's office for Biogen Region Europe and Canada. Previously at Biogen he also served as a Director in Global Neurology for the natalizumab program in Cambridge (MA, USA). Prior to joining industry, he was a Clinical Professor of Neurology at the University of Liège in Belgium. Shibeshih completed neurology postgraduate training at the University of Liège and has a PhD in Biomedical Science in the field of Developmental Neurobiology. Shibeshih has been a post-doctoral fellow in the Laboratory of Cellular and Synaptic Neurophysiology at the National Institutes of Health (Bethesda, MD, USA) and later at the Center for Neuroscience Research of Children's National Medical Center in Washington DC. $\mathrm{He}$ is a member of the Belgian Neurological Society.
\end{abstract}

First draft submitted: 27 October 2017; Published online: 5 January 2018

Can you introduce yourself \& give us an overview of your role?

I am a neurologist and neurobiologist by background, and am now International Medical Director, in Global Product Development Medical Affairs at Roche (Basel, Switzerland). I am completely dedicated to ocrelizumab (OCR) research, and in particular the exploratory analyses of the Phase III trials to attempt to further advance our understanding of multiple sclerosis (MS), with a special interest for the mechanisms of the underlying progression, which we are uncovering by assessing OCR in relapsing and primary progressive forms of MS (RMS and PPMS).

Can you tell us more about the data presented at ECTRIMS \& the use of progression independent of relapse activity (PIRA) as an end point?

A poster was presented at ECTRIMS that included the development of a new outcome measure as an end point for patients with relapsing forms of MS, called composite progression independent of relapse activity (or composite PIRA) [1]. Composite PIRA enables us to disentangle the progression of disability that is independent of relapse activity - patients with relapsing forms of MS may experience relapses from time to time, but in between these relapses they may experience an insidious underlying progression. Composite PIRA is about measuring that aspect of the worsening of patients in terms of disability not influenced by acute relapses. It is also a comprehensive measure of disability, and includes three components:

- Conventional Expanded Disability Status Scale (EDSS);

- An ambulation measure, the timed 25-foot walk (T25FW);

- A measure of upper extremity function and manual dexterity, called the nine-hole peg test (9HPT).

These three components provide a comprehensive assessment of disability, and the methods used herein where we re-baselined the assessments after each relapse enabled us to extract from overall disability the part that is happening independently from the occurrence and impact of relapses. It was demonstrated that OCR (compared with IFN $\beta 1 \mathrm{a} 44 \mu \mathrm{g}$ ) reduced the risk of experiencing those events of PIRA in patients with RMS, by 25 and $23 \%$ for the 12 - and 24 -week confirmed events, respectively. And possibly more importantly, when you look

Future $\because \cdots$ Medicine 
beyond the intention-to-treat (ITT) population of the OPERA I and OPERA II trials, and perform the analysis on those RMS patients who are likely to be at a higher risk of progressive MS disease course, OCR reduced the risk of 12- and 24-week-confirmed PIRA events by $40 \%$ and 36\%, respectively. This is an important aspect of the exploratory understanding of the efficacy of OCR, and completes the evolving demonstration of MS disease course as a continuum where progressive aspects of the pathology appear to be present already in early RMS at a sizeable extent.

You also presented research on the development of a potential measure to look at underlying disease activity in the brain using MRI; could you tell us more about this?

In parallel to the work on PIRA, research has been conducted in collaboration with NeuroRx Research (Montreal, QC, Canada) to measure an imaging component of chronic disease activity in PPMS and RMS, called slowly evolving lesions (SELs) [2]. This work uses conventional brain MRIs as acquired in clinical practice - T1- and T2weighted imaging. SELs are pre-existing and chronically active lesions that are characterized by local expansion and ongoing accumulation of tissue damage, which occurs independently from acute inflammation that is traditionally measured by gadolinium enhancement. This helps us, together with the PIRA metric, to further characterize the scope and magnitude of chronic disease activity in MS, which, without these techniques, might continue to go unnoticed. We also demonstrated that SELs are not only present in PPMS, but also in RMS patients. Whether ongoing brain tissue damage associated with SELs may reflect mostly microglia/macrophage-mediated chronic inflammation and/or secondary Wallerian neurodegeneration within lesions of MS patients remains to be investigated.

\section{How will these data affect patients with MS?}

This consolidates the evidence that MS can be seen as a single disease 'continuum', that is, an array of entities ordered between opposite ends, and sharing common properties to various extents with temporal fluctuations within and between patients longitudinally. This work is bringing consistency to the demonstration of efficacy of OCR along the continuum, but importantly it is also advancing the way we understand MS, which is a prerequisite to optimize disease control and halt disability accumulation.

As of the end of October 2017, we had approximately 20,000 patients that have been treated with OCR worldwide, when you combine use both within and outside clinical trials. We are committed to rapidly bringing this drug to patients worldwide because MS is a progressive disease where time matters; the median time from a patient being diagnosed with PPMS, to becoming wheelchair-bound, is approximately 12-13 years [3], so making a treatment available for those patients when they are first diagnosed is definitely a crucial priority for Roche.

\section{What other work is Roche doing in the area of monitoring MS disease progression?}

Roche is also making tremendous efforts to transpose the knowledge we have gained into clinical care. With the prior knowledge that a lot of the disability accumulation in MS might have been going unnoticed in the clinical trial setting, it is likely that this is also true in the daily life of patients. The question then becomes, how do you transform the paradigm of the way we measure MS, so that when patients see their physician, perhaps every 6-12 months, they are actually in possession of data that may accurately characterize how they have been in between?

In this respect, we presented the interim results of a proof-of-concept study called FLOODLIGHT (NCT02952911), aimed at advancing the use of smartphone sensor-based technology to develop more sensitive, specific and continuous measures of patient symptoms [4]. The FLOODLIGHT program and the ongoing proof-of-concept study is currently taking place in two centers in Barcelona and San Francisco, and has recruited $76 \mathrm{MS}$ patients and 25 healthy controls. These participants have been asked to use a smartphone on a daily basis to perform a very short battery of active tests, taking them around 5-7 min, alongside accepting to be continuously monitored via the device. This allows us to capture huge amounts of information, passively and continuously for instance from the accelerometer, gyroscope and GPS technology in the smartphone. We are around two-thirds of the way through this 6-month trial, and have already collected an enormous quantity of sensor-based data.

The FLOODLIGHT monitoring solution measures three domains, including cognition with a simple information processing speed test; hand/arm function; and ambulation or mobility as a whole. The data presented at ECTRIMS focused on hand/arm function and ambulation, and we found that we were able to collect more granular data using the smartphone sensors than could have been detected in clinic visits. For example, in classic in-clinic visits for an MS patient, manual dexterity is monitored by a physician timing how long time it takes for 
the patient to put nine pegs into nine holes, and then removing them (the 9HPT). This provides a single piece of time information, such as, for example 25 or $30 \mathrm{~s}$, but ignores the quality of the task and how those patients have actually used their hands during the test.

Using the smartphone, we conduct an activity that is called the pinching test, which asks patients to squeeze tomatoes that are randomly moving around on the phone's touchscreen for $30 \mathrm{~s}$. Not only can the device measure the number of tomatoes that are squeezed, which could translate into the number of 'bottles of ketchup' that are filled at the end of the week (a fun metric for the patient!), but can measure all the information of the contact of the finger of the patient with the touchscreen at every pinching attempt, as well as the movement of the device held by the 'untested' hand. What is clear, for instance, is that the velocity of the task on the touchscreen, such as the time between two consecutive pinching actions, is abnormal in MS patients compared with healthy controls, which would be expected; but beyond that what we see is when we take those MS patients who have had normal performances according to their in-clinic conventional 9HPT and assess those patients for some of the manifold pinching parameters, we see that they are actually doing worse than the healthy controls. This suggests that those sensitive sensor-based outcomes are capable of discriminating MS-related abnormalities of the manual dexterity that conventional in-clinic tests may miss, and sometimes that the patient may not consciously experience.

A similar situation can be shown with ambulation. For ambulation there are three tests in the FLOODLIGHT program: a static balance test; a so-called 2-min walking test where patients are asked to walk as fast as possible for $2 \mathrm{~min}$; and the one we are the most excited about so far, which is called the U-turn test. In the U-turn test patients are asked to do at least five U-turns while walking comfortably. It takes $1 \mathrm{~min}$ and enables us to capture all the sensor information not only while they walk, but while they turn, which represents an exquisitely challenging task for bipedal humans that we tend to ignore. Then, using this active testing paradigm, we have developed an algorithm that is able to detect when the patient turns at other times, outside of the active test battery, via the passive, continuous monitoring of the device. We use machine learning techniques to learn from the active test how patients are turning, and have developed techniques that will enable the capture of turns that are happening in their daily routine. We then measure not only how frequently they turn, but how they turn. This is important, as when ambulation quality is subtly reduced, it is not necessarily how fast the patient walks, or how many steps they take in a day (information that is also captured by their device), but how they are walking that could be the most sensitive metric to detect change in gait ataxia relating to the disease.

As an example, in the study we had a patient who experienced symptoms that resulted in them picking the options 'I am doing worse' and 'I consider I experience a relapse' in the symptom tracker on their device. But interestingly, at that patient's next in-clinic visit, they had no recollection of this, and so did not report the relapse in the classical, in-clinic clinical trial visit. However, as we had the information from the symptom tracker, we could reconstruct the history from the active and passive monitoring of that particular patient, and demonstrate that their U-turn behavior actually changed significantly at the time the patient checked that box and subsequently. So, this is a demonstration that sensor-based outcomes collected from a smartphone not only empower the patient to self-monitor their condition, it also creates a kind of augmented reality of the clinical picture of MS, and certainly of the subjective but very real experience of the patient living with the disease.

\section{Is there any final information you'd like to pass on to the readers of Neurodegenerative Disease Management?}

The findings from FLOODLIGHT complement what we are doing in our clinical trials, which are all about advancing the way we understand MS and the way MS may evolve, probably more insidiously than previously detected with conventional tools. And importantly, if you consider that the majority of progression in RMS patients is not due to relapses, then the real challenge is about how to measure that, and ensure this does not go unnoticed, and that it can be treated adequately with molecules that impact that particular aspect of MS pathology.

There is probably nothing more predictive of the long-term outcome of MS than 'progression' - a patient who is progressing is likely to continue to progress. When you think about this, and the fact that diagnosis can occur in patients that are 20-30 years of age, then it is clear that time matters. One may acknowledge that at present, aspects of the disease are missed, especially at early stages due to the lack of proper measures in clinical trials and certainly in routine care. Now that we have treatment options, such as OCR, for the progressive disease course in its primary-onset form, it is crucially important that we have measures in place to detect and monitor these aspects. 


\section{Acknowledgements}

The opinions expressed in this interview are those of S Belachew and do not necessarily reflect the views of Future Medicine Ltd.

\section{Financial \& competing interests disclosure}

$\mathrm{S}$ Belachew is an employee and shareholder of F Hoffmann-La Roche Ltd (Basel, Switzerland). The interviewee has no other relevant affiliations or financial involvement with any organization or entity with a financial interest in or financial conflict with the subject matter or materials discussed in the manuscript apart from those disclosed.

No writing assistance was utilized in the production of this manuscript.

\section{References}

1. Kappos L, Wolinsky JS, Giovannoni G et al. Ocrelizumab reduces disability progression independent of relapse activity in patients with relapsing multiple sclerosis. Presented at: The 7th Joint ECTRIMS-ACTRIMS Meeting. Paris, France, 25-28 October 2017. https://onlinelibrary.ectrims-congress.eu/ectrims/2017/ACTRIMS-ECTRIMS2017/200309/ludwig.kappos.ocrelizumab.reduces. disability.progression.independent.of.html

2. Elliott C, Wolinsky JS, Hauser SL et al. Detection and characterization of slowly evolving lesions in multiple sclerosis using conventional brain MRI. Presented at: The 7th Joint ECTRIMS-ACTRIMS Meeting. Paris, France, 25-28 October 2017.

https://onlinelibrary.ectrims-congress.eu/ectrims/2017/ACTRIMS-ECTRIMS2017/202544/colm.elliott.detection.and.characterisation. of.slowly.evolving.lesions.in.html

3. Confavreux C, Vukusic S. Natural history of multiple sclerosis: a unifying concept. Brain 129(Pt 3), 606-616 (2006).

4. Mulero P, Midaglia L, Montalban X et al. Interim analysis from FLOODLIGHT: a prospective pilot study to evaluate the feasibility of conducting remote patient monitoring with the use of digital technology in patients with multiple sclerosis. Presented at: The 7th Joint ECTRIMS-ACTRIMS Meeting. Paris, France, 25-28 October 2017. http://onlinelibrary.ectrims-congress.eu/ectrims/2017/ACTRIMSECTRIMS2017/200881/patricia.mulero.interim.analysis.from.floodlight.a.prospective.pilot.study.to.html 